\title{
Performance Analysis of the Enhanced Distributed Coordination Function in the IEEE 802.11e
}

\author{
Yu-Liang Kuo*, Chi-Hung Lu*, Eric Hsiao-Kuang $\mathrm{Wu}^{\dagger}$, Gen-Huey Chen*, and Yi-Hsien Tseng* \\ * Dept. of Computer Science and Information Engineering, National Taiwan University, Taipei, Taiwan, R.O.C. \\ ${ }^{\dagger}$ Dept. of Computer Science and Information Engineering, National Central University, Chung-Li, Taiwan, R.O.C.
}

\begin{abstract}
Recently, the IEEE 802.11 Task Group E is standardizing a distributed access approach, called the enhanced distributed coordination function (EDCF), to support service differentiation in WLANs. In EDCF, service differentiation is achieved by assigning different values of EDCF access parameters (e.g., the minimum contention window, the maximum contention window, and the arbitration interframe space) to different traffic classes. In order to satisfy specific QoS requirements for different traffic classes, a mathematical analysis turns to be helpful to analyze the system performance. In this paper, we provide an analytical model to study the expected bandwidth for each traffic class in EDCF. We validate the accuracy of the model by using the $n s-2$ simulator. Some performance evaluations are demonstrated in comparison with the IEEE 802.11 legacy MAC protocol. The model can be also used as guidelines for admission control or resource management schemes.
\end{abstract}

\section{INTRODUCTION}

There is an increasing demand that traffic classes with different QoS requirements will be provided in future WLANs. Since these traffic classes require distinct specific features, such as delay-sensitivity or bandwidth requirement, it is desired to provide a service differentiation mechanism in the IEEE 802.11 standard [3]. In order to provide service differentiation in a wireless environment, the MAC protocol is required to support some degree of separation between different traffic classes. Recently, the IEEE 802.11 Task Group $\mathrm{E}$ (IEEE 802.11e) is specifying a distributed access approach, called EDCF, to support service differentiation in the MAC layer [5]. In this paper, we use an analytical model to study the performance of EDCF, which is in comparison with the IEEE 802.11 legacy MAC.

The fundamental access method in IEEE 802.11 is distributed coordination function (DCF), which is based on carrier sense multiple access with collision avoidance (CSMA/CA) protocol. A mobile station that intends to transmit a packet waits until the channel is sensed idle for a time period equal to the DCF interframe space (DIFS). If the channel is sensed idle for a duration of DIFS, then it can immediately transmit a packet. Otherwise, the mobile station will generate a backoff time counter. A discrete time counter is used and the time following an idle DIFS is slotted. A mobile station is allowed to transmit only at the beginning of a time slot.

When a mobile station senses the channel busy during the duration of DIFS, the backoff time counter is randomly selected from the range $(0, \mathrm{CW})$, where $\mathrm{CW}$ is called the contention window. At the first transmitting attempt, $\mathrm{CW}$ is assigned the value $\mathrm{CW}_{\min }$, which is called the minimum contention window. In the consecutive unsuccessful transmissions (due to collisions), the value of $\mathrm{CW}$ is increasing up to the maximum value $\mathrm{CW}_{\max }=2^{m} \mathrm{CW}_{\text {min }}$, where $m$ is called the maximum backoff stage.

The backoff time counter is decreased as the channel is sensed idle and suspended as the channel is sensed busy. After the suspension, the counter is reactivated as the channel is again sensed idle for a duration of DIFS. The mobile station will transmit a packet when the counter reaches zero.

The EDCF introduces the concept of access categories (ACs), which are variants of the DCF access mechanism. Different ACs uses different values of arbitration interframe space duration (AIFSD), minimum contention window $\left(\mathrm{CW}_{\min }\right)$, and minimum contention window $\left(\mathrm{CW}_{\max }\right)$. Traffic classes with smaller values of $\mathrm{CW}_{\min }$ and $\mathrm{CW}_{\max }$ yield higher priorities. Furthermore, different interframe spaces can be used by different traffic classes. DCF Interframe space (DIFS) is substituted for the AIFSD. AIFSD is at least a duration of short interframe space (SIFS) plus a slot time and can be enlarged individually by different traffic classes. AIFSD can be computed as the following:

$$
\text { AIFSD }=\text { SIFS }+ \text { AIFS } \times \text { aSlotTime, }
$$

where AIFS is a positive integer and greater than 1. Hence AIFSD is determined by AIFS and traffic classes with smaller values of AIFS yield higher priorities. These three parameters are gathered and called a QoS parameter set.

In EDCF, data packets are delivered through multiple backoff instances within one mobile station. A single mobile station may implement up to 4 transmission queues and each transmission queue use a specific $\mathrm{AC}$ for contending the channel access, as depicted in Fig. 1. The IEEE 802.11e draft specifies four default ACs, which are shown in Table I. Each queue within the mobile station contends for the channel access and independently starts its backoff depending on its associated AC. If the backoff time counters of two or more parallel queues within a single mobile station reach zero at the same time, an internal scheduler will resolve the internal collision. The scheduler grants the channel access to the queue in terms of its particular scheduling algorithm.

In this paper, we propose an analytical model for the EDCF access mechanism to evaluate expected bandwidth of each traffic class. A mathematical model is helpful to obtain the performance analysis and suggest some guidelines 


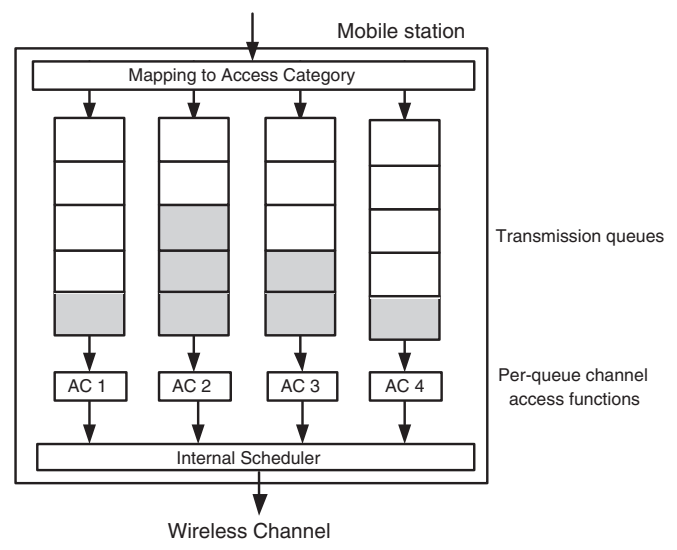

Fig. 1. A single mobile station can implement up to 4 transmission queues. Each queue is mapping to a particular access category.

TABLE I

The Four Default ACs

\begin{tabular}{ccccc}
\hline AC & AIFS & $\mathrm{CW}_{\min }$ & $\mathrm{CW}_{\max }(m)$ & Designation \\
\hline 0 & $34 \mu \mathrm{s}$ & 15 & $1023(6)$ & Best Effort \\
1 & $25 \mu \mathrm{s}$ & 15 & $1023(6)$ & Video Probe \\
2 & $25 \mu \mathrm{s}$ & 7 & $15(1)$ & Video \\
3 & $25 \mu \mathrm{s}$ & 3 & $7(1)$ & Voice \\
\hline
\end{tabular}

for admission control or resource management schemes. By using $n s-2$ simulator [1], we validate the analytical model with two simple scenarios. The validation shows that the analytical model approximately coincides with simulative one. Some performance evaluations will be also provided in the subsequent section.

The paper is organized as follows. Section II provide an analytical model for EDCF to study the expected bandwidth and expected packet delay of each traffic class. The model validation is demonstrated in Section III. Section IV presents numerical results, in comparison with DCF. The conclusion is drawn in Section V.

\section{Analytical Model}

The environment we consider is a single wireless cell coordinated by an AP. In a single cell environment, each mobile station which intends to transmit a packet needs to forward its packet to the corresponding AP. The transmitted packets should be forwarded to the AP even if the packets are destined to the mobile station in the same cell. We assume all mobile stations and the corresponding AP can communicate with each other without obstacle and the air condition is assumed to be error-free. The access mechanism we consider is a fourway handshaking protocol by using the RTS/CTS/DATA/ACK dialogue.

Without loss of generality, we assume there are $K$ traffic classes with distinct QoS requirements in the system. Traffic class $k$ stations use $\mathrm{AC}_{k}$ to access the wireless channel. Specifically, there are $\mathbf{n}=\left(n_{0}, n_{2}, \ldots, n_{K-1}\right)$ mobile stations, where $n_{k}(0 \leq k \leq K-1)$ is the number of mobile stations which generate traffic class $k$ packets. For convenience, let the traffic class $k$ packet be denoted by class- $k$ packet and the class- $k$ station is referred to as the mobile station that generates class- $k$ packets to transmit. We assume that each class- $k$ packet has constant length $L_{k}$ and requires $\frac{L_{k}}{M}$ seconds for transmission delay, where $M$ is the average channel bit rate. We also assume that each mobile station always has a packet ready to transmit. In other words, we consider the saturation condition [2]. The maximum propagation delay for all packets between two mobile stations within the cell is assumed to be a constant length of $\tau$ seconds.

At each transmission attempt, we assume that each class$k$ packet $(0 \leq k \leq K-1)$ has common probability $p_{k}$ of involving in collision, independent of retransmission history. The assumption of $p_{k}$ is originated from Bianchi's model [2] but we extend the assumption to allow $K$ traffic classes. Suppose that a class-k station that has involved in $j$ times of collision will select the backoff time counter $B_{k_{j}}(j \geq 0)$ to decrement before (re)transmission. Let $J_{k}$ denote the number of collision a class-k station has suffered. As a class-k station intends to transmit a packet, the expected backoff time counter $E\left[B_{k}\right]$ can be computed by conditioning on $J_{k}$

$$
E\left[B_{k}\right]=\sum_{i=0}^{\infty} E\left[B_{k_{i}}\right] P\left\{J_{k}=i\right\} .
$$

The distribution of $J_{k}$ is

$$
P\left\{J_{k}=i\right\}= \begin{cases}p_{k}^{i} & \text { if } i \geq 1, \\ 1-\sum_{j=1}^{\infty} p_{k}^{j} & \text { if } i=0 .\end{cases}
$$

Let $W_{k}, \mathrm{AIFS}_{k}$, and $m_{k}$ be the minimum contention window, AIFS, and maximum backoff stage for each class$k$ station. Since $B_{k_{j}}$ is selected from the current contention window size (the current contention window size is obtained according to the binary exponential backoff procedure) in a uniform way, its distribution is

$$
\begin{gathered}
P\left\{B_{k_{j}}=i\right\}=\frac{1}{2^{\beta_{k}(j)}\left(\mathrm{W}_{k}+1\right)+\mathrm{AIFS}_{k}}, \\
i=0,1, \cdots, 2^{\beta_{k}(j)}\left(\mathrm{W}_{k}+1\right)+\mathrm{AIFS}_{k}-1,
\end{gathered}
$$

where $\beta(\cdot)$ is defined as the following:

$$
\beta_{k}(i)= \begin{cases}i & \text { if } 0 \leq i \leq m_{k}-1, \\ m_{k} & \text { if } i \geq m_{k} .\end{cases}
$$

The expectation of $B_{k_{j}}$ can be easily derived from (3)

$$
E\left[B_{k_{j}}\right]=\frac{2^{\beta_{k}(j)}\left(\mathrm{W}_{k}+1\right)+\mathrm{AIFS}_{k}-1}{2} .
$$

According to (2) and (4), (1) can be expressed as

$$
\begin{aligned}
E\left[B_{k}\right] & =\left(1-\sum_{i=1}^{\infty} p_{k}^{i}\right) \frac{W_{k}+\mathrm{AIFS}_{k}}{2} \\
& +\sum_{i=1}^{\infty} p_{k}^{i} \frac{2^{\beta_{k}\left(J_{k}\right)}\left(\mathrm{W}_{k}+1\right)+\mathrm{AIFS}_{k}-1}{2}
\end{aligned}
$$




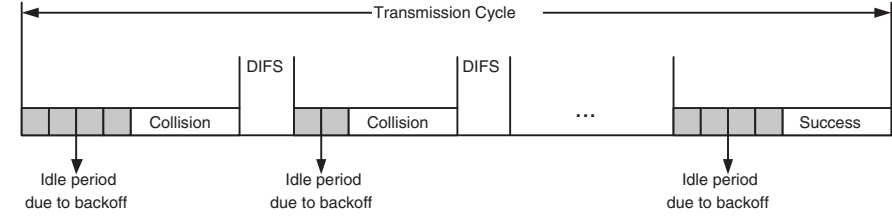

Fig. 2. A single mobile station can implement up to 4 transmission queues. Each queue is mapping to a particular access category.

At a given time slot, The probability that a class- $k$ station will transmit is

$$
q_{k}=\frac{1}{E\left[B_{k}\right]}
$$

The collision probability for a class- $k$ packet transmission can be computed as

$$
p_{k}=\left(1-\prod_{j=0, j \neq k}^{K-1}\left(1-q_{j}\right)^{n_{j}}\left(1-q_{k}\right)^{n_{k}-1}\right) .
$$

By observing the transmission behavior in the wireless channel, a pattern of periodical cycles can be found. Each cycle, named as transmission cycle, consists of some idle periods, some unsuccessful periods (due to collisions), and a successful period, as depicted in Fig. 2. When each cycle ends in a successful period, a consecutive cycle will restart with respect to the ordered sequences (idle periods, unsuccessful periods, a successful period). Let $E[I], E[C]$, and $E[S]$ be the sum of the expected lengths of all idle periods, all unsuccessful periods, and the expected length of a successful period, respectively. Also, let $E\left[L_{k}\right]$ be the expected number of bits a class- $k$ packet will be transmitted in a successful period. As a result, using renewal theory arguments [6][7], the expected bandwidth for each class- $k$ station $(0 \leq k \leq K-1)$ is given as

$$
\rho_{k}=\frac{E\left[L_{k}\right]}{E[I]+E[C]+E[S]}
$$

The probability that a class-k packet will be transmitted in a transmission cycle is

$\gamma_{k}=\mathrm{P}\{$ Transmitting station $=1$ and is class $-k$ station

$\mid$ Transmitting station $\geq 1\}$

Hence (9) can be computed as

$$
\gamma_{k}=\frac{n_{k} q_{k}\left(1-q_{k}\right)^{n_{k}-1} \prod_{i=0, i \neq k}^{K-1}\left(1-q_{i}\right)^{n_{i}}}{1-\prod_{i=0, i \neq k}^{K-1}\left(1-q_{i}\right)^{n_{i}}} .
$$

Since only one particular class- $k$ packet $(0 \leq k \leq K-1)$ can be transmitted in a successful period, we compute $E\left[L_{k}\right]$ according to the normalized probability $\gamma_{k}$. Thus, the expected number of bits a class- $k$ packet transmitted in a successful period is given as

$$
E\left[L_{k}\right]=\frac{\gamma_{k}}{\gamma_{0}+\gamma_{1}+\cdots+\gamma_{K-1}} L_{k}
$$

Also the expected AIFS a class-k packet required in a successful period is given as

$$
E\left[\mathrm{AIFS}_{k}\right]=\frac{\gamma_{k}}{\gamma_{0}+\gamma_{1}+\cdots+\gamma_{K-1}} \operatorname{AIFS}_{k} .
$$

The expected number of bits transmitted in a successful period is $E[L]=\sum_{k=0}^{K-1} E\left[L_{k}\right]$ and the expected AIFS required in a successful period is $E[A]=\sum_{k=0}^{K-1} E\left[\mathrm{AIFS}_{k}\right]$.

Let $T_{S I F S}, T_{A C K}, T_{R T S}, T_{C T S}$, and $H$ be the duration required for a SIFS, transmitting a ACK, transmitting a RTS, transmitting a CTS, and transmitting a PHY/MAC header, respectively. The expected length of a successful period in a transmission cycle is approximately expressed as

$E[S]=T_{R T S}+T_{C T S}+T_{A C K}+\frac{E[L]}{M}+4 \tau+4 T_{S I F S}+E[A]+H$.

Given the number of each traffic flow $\mathbf{n}$, we let $N_{c}$ be the random variable representing the number of colliding periods needed in a transmission cycle. The distribution of $N_{c}$ is geometric with parameter $\left(1-p_{c}\right)$, where $p_{c}$ is the collision probability in the channel and can be computed as

$$
\begin{aligned}
& \mathrm{P}\{\text { Transmitting station } \geq 2 \\
& \mid \text { Transmitting station } \geq 1\}
\end{aligned}
$$

From (14), $p_{c}$ can be computed as

$p_{c}=\frac{1-\prod_{i=1, i \neq k}^{K}\left(1-q_{i}\right)^{n_{i}}-\sum_{i=1}^{K} n_{i} q_{i}\left(1-q_{i}\right)^{n_{i}-1} \prod_{j=1, j \neq i}^{K}\left(1-q_{j}\right)}{1-\prod_{i=1}^{K}\left(1-q_{i}\right)^{n_{i}}}$

As a result, we have

$$
E\left[N_{c}\right]=\frac{p_{c}}{1-p_{c}}
$$

The number of time slots required for an idle period in a transmission cycle can be viewed as a geometric random variable with parameter $\left(1-\prod_{i=1}^{K}\left(1-q_{i}\right)^{n_{i}}\right)$. Hence, the expected length of an idle period can be computed as

$$
\begin{aligned}
\delta \cdot \sum_{j=0}^{\infty} j \cdot\left(1-\prod_{i=1}^{K}\left(1-q_{i}\right)^{n_{i}}\right) \cdot\left(\prod_{i=1}^{K}\left(1-q_{i}\right)^{n_{i}}\right)^{j} . \\
=\left(\delta \cdot \frac{\prod_{i=1}^{K}\left(1-q_{i}\right)^{n_{i}}}{1-\prod_{i=1}^{K}\left(1-q_{i}\right)^{n_{i}}}\right) .
\end{aligned}
$$

Since the lengths of all idle periods are assumed to be identical and independent and there are $\bar{N}_{c}+1$ idle periods in a transmission cycle, the sum of the expected lengths of all idle periods in a transmission cycle is

$$
E[I]=\left(E\left[N_{c}\right]+1\right)\left(\delta \frac{\prod_{i=1}^{K}\left(1-q_{i}\right)^{n_{i}}}{1-\prod_{i=1}^{K}\left(1-q_{i}\right)^{n_{i}}}\right) .
$$

The sum of the expected lengths of all unsuccessful periods in a transmission cycle is

$$
E[C]=E\left[N_{c}\right]\left(\tau+T_{S I F S}+E[A]+T_{R T S}+H\right) .
$$

This follows since the length of an unsuccessful period is $\tau+T_{S I F S}+E[A]+T_{R T S}+H$ and the expected number of unsuccessful periods is $E\left[N_{c}\right]$.

From(11), (13), (18), and (19), we can express the expected bandwidth of each traffic class in closed-forms. 
TABLE II

THE VALUES OF PARAMETERS USED IN MODEL VALIDATION

\begin{tabular}{cc}
\hline FTP packet payload size $\left(\mathrm{AC}_{0}\right)$ & 1500 bytes \\
Video packet payload size $\left(\mathrm{AC}_{2}\right)$ & 1464 bytes \\
Voice packet payload size $\left(\mathrm{AC}_{3}\right)$ & 96 bytes \\
PHY header & 6 bytes \\
MAC header & 34 bytes \\
RTS & 20 bytes \\
CTS & 14 bytes \\
ACK & 14 bytes \\
Propagation delay & $1 \mu \mathrm{s}$ \\
Average channel bit rate & $1 \mathrm{Mbps}$ \\
SIFS & $16 \mu \mathrm{s}$ \\
Slot time & $9 \mu \mathrm{s}$ \\
\hline
\end{tabular}

\section{Model Validation AND Numerical Results}

In this section, we present the model validation and numerical results. By using the $n s-2$ simulator, we run two scenarios and compare the analytical model with the simulative one. The values of parameters used in the analytical and simulative model are summarized in Table II. In this validation, we have constant packet payload size, as shown in Table II. We have three usual applications (FTP, video, and voice application) and each one is associated with its default $\mathrm{AC}$ for contending the channel access. The values of parameters is assigned depending on the IEEE 802.11a specification [], excepting the average channel bit rate, which is assumed to be 1 Mbps.

\section{A. Model Validation}

In the first scenario, we consider a simple one that contains three flows, which is associated with $\mathrm{AC}_{0}, \mathrm{AC}_{2}$, and $\mathrm{AC}_{3}$ for contending the channel access. Each flow is destined to the AP and the AP is destined to the corresponding host in the Internet. As a result, we have $K=3$ and $\mathbf{n}=$ $(1,1,1)$. Fig. 3(a) shows that the simulative model, in which diamond line represents simulative bandwidth of video, square line represents simulative bandwidth of FTP, and circle line represents simulative bandwidth of audio, is close to the expected bandwidth made by analytical model.

In the second scenario, we consider more traffic flows. We add two stations to the first scenario, in which one station runs voice application and another one runs video applications. As a result, we have $K=3$ and $\mathbf{n}=(1,2,2)$. Fig. 3(b) shows that the simulative model are also close to the expected bandwidth made by analytical model.

\section{B. Numerical Results}

In this subsection, we show the effect of EDCF and performance the collision and transmission probability of each traffic class as the number of traffic flows increases. Since the voice application has higher priority than the video and FTP applications, it can tolerate the increment of FTP applications, as show in Fig. 4(a). In Fig. 4(a), the bandwidth of video application (dotted line) is decreasing as the number of FTP flows increases but the effect of decrement is getting stable. Although the bandwidth of voice application is decreasing as the number of FTP flows increases, the extent of decrement is smaller than video application. Fig. 4(a) also suggests that the admission control should consider the best-effort traffic since the video is decreasing from $675 \mathrm{Kbps}$ to $210 \mathrm{Kbps}$. Instead of increasing the number of FTP flows, Fig 4(b) shows the change of expected bandwidth of the FTP and video applications as the voice application is increasing. Since the voice application has higher priority than the video and FTP applications, the bandwidth of the FTP and video will be decreasing dramatically as the number of the voice flows increases. Fig 4(a) and Fig. 4(b) also indicate that EDCF can achieve the service differentiation.

Considering the collision and transmission probabilities, Fig. 5(a) shows that the change of both probabilities as the number of FTP flows increases. From Fig. 5(a), we can see that the effect of the number of FTP flows is small. However, in Fig. 5(b), both probabilities are getting down as the number of voice flows increases. These plots can also indicate that EDCF can achieve the service differentiation.

\section{CONCLUSION}

In this paper, we introduced an approximate analytical model to evaluate the expected bandwidth of each traffic class for the service differentiation mechanism based on EDCF. By using the $n s-2$ simulator, we use two scenarios to validate the model is accurate. The numerical results also indicate that EDCF can achieve the service differentiation. In the future work, the expected packet delay of each traffic class can be approximately derived. With this analytical model, some admission control and resource management schemes can be also design to meet the QoS.

\section{ACKNOWLEDGMENT}

This work was supported in part by the MediaTek Inc. This work was also supported by the Communications Software Technology project of Institute for Information Industry and sponsored by MOEA, R.O.C.

\section{REFERENCES}

[1] "The Network Simulator - ns-2," http://www.isi.edu/nsnam/ns/.

[2] G. Bianchi, Performance Analysis of the IEEE 802.11 Distributed Coordination Function, IEEE Journal of Selected Areas on Communication 18(3) (March 2000) 535-547.

[3] Wireless LAN Medium Access Control (MAC) and Physical Layer (PHY) Specification, IEEE 802.11 WG, Aug. 1999.

[4] Wireless LAN Medium Access Control (MAC) and Physical Layer (PHY) Specifications: High-speed Physical Layer in the 5GHz Band, IEEE Std 802.11a-1999, Sep. 1999.

[5] Wireless LAN Medium Access Control (MAC) and Physical Layer (PHY) Specification: Medium Access Control (MAC) Enhancements for Quality of Service $(Q o S)$, IEEE Std. 802.11e/D4.0, Nov. 2002.

[6] L. Kleinrock, Queueing Systems, Vol, I: Theory (Wiley-Interscience, New York, NY, 1975)

[7] L. Kleinrock and F. Tobagi, "Packet switching in radio channels: Part I - carrier sense multiple-access modes and their throughput-delay characteristics," IEEE Trans. Commun., vol. com-23, no. 12, December 1975. 


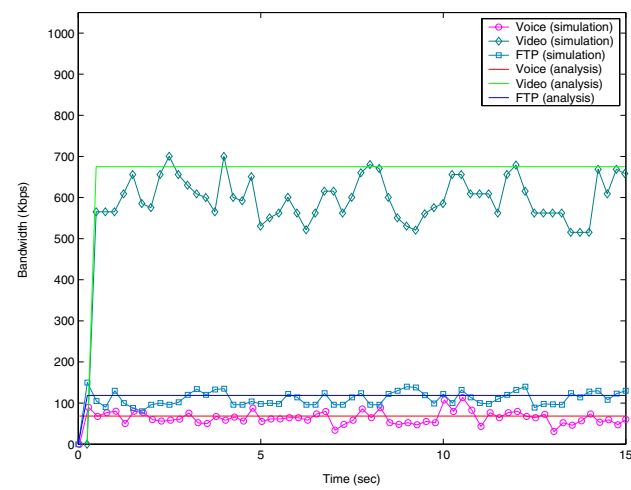

(a) The first scenario. (one FTP, one video, and one voice flows).

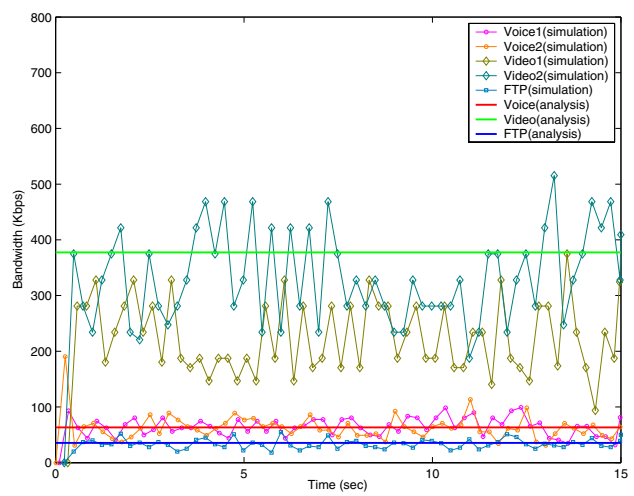

(b) The second scenario. (one FTP, two video, and two voice flows).

Fig. 3. Model validation: simulative model vs. analytical model.

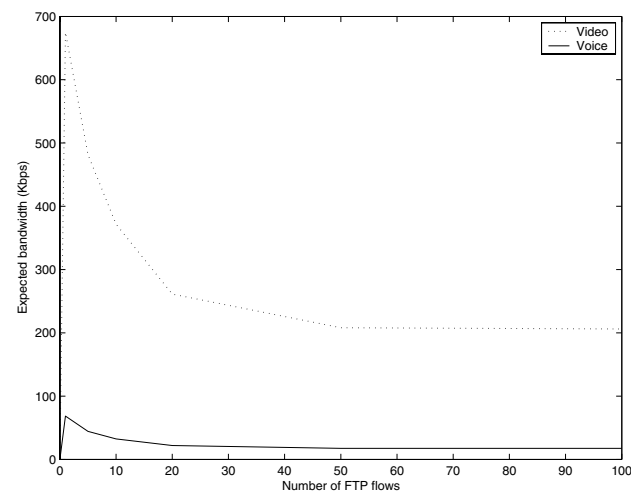

(a) The effect of expected bandwidth as the number of FTP flows increases.

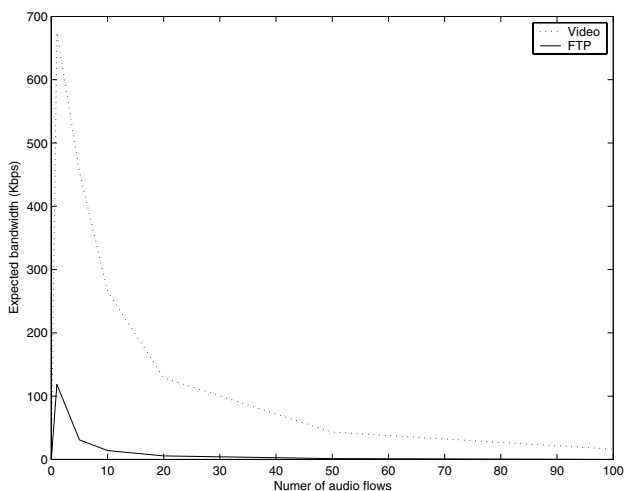

(b) The effect of expected bandwidth as the number of voice flows increases.

Fig. 4. The effect of expected bandwidth.

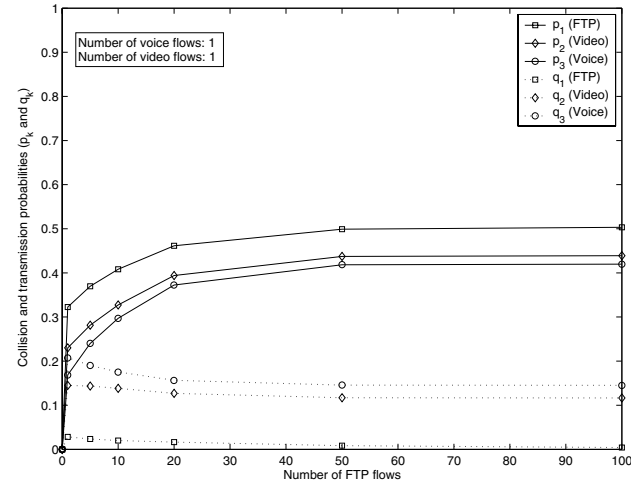

(a) The effect of collision and transmission probabilities as the number of FTP flows increases.

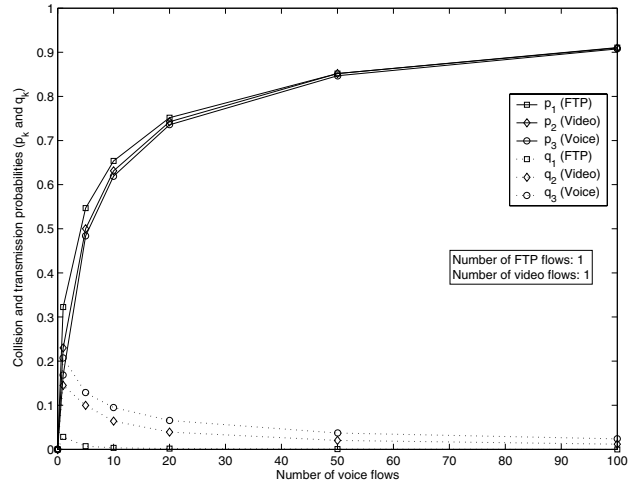

(b) The effect of collision and transmission probabilities as the number of voice flows increases.

Fig. 5. The effect of collision and transmission probabilities. 